\title{
Drivers of the Peaking and Decoupling Between CO2 Emissions and Economic Growth Around 2030 in China
}

Weifeng Gong ( $\sim$ gongweifeng@qufu.edu.cn )

Qufu Normal University

Chuanhui wang

Qufu Normal University https://orcid.org/0000-0002-3559-9813

Zhenyue Fan

Qufu Normal University

Yang Xu

Ocean University of China

\section{Research Article}

Keywords: carbon emissions, peaking, elastic decoupling, factor decomposition, Markov chain

Posted Date: June 2nd, 2021

DOI: https://doi.org/10.21203/rs.3.rs-520919/v1

License: (c) This work is licensed under a Creative Commons Attribution 4.0 International License.

Read Full License

Version of Record: A version of this preprint was published at Environmental Science and Pollution Research on August 16th, 2021. See the published version at https://doi.org/10.1007/s11356-021-155186. 
1 Drivers of the Peaking and Decoupling between $\mathrm{CO}_{2}$ Emissions

2 and Economic Growth around 2030 in China

3 Weifeng Gong ${ }^{1,2}$, Chuanhui Wang ${ }^{1,2}$, Zhenyue Fan ${ }^{1}$, Yang Xu ${ }^{3}$

$4 \quad{ }^{1 .}$ School of Economics, Qufu Normal University, Rizhao 276826, China

$5{ }^{2}$ School of Economics and Management, Nanjing University of Aeronautics and Astronautics Jiangsu, 211006, China

$6 \quad{ }^{3 .}$ Management College, Ocean University of China, Qindao, 266100, China

7 Abstract: Reaching the peak of carbon dioxide emissions is the basis and premise of carbon neutrality. In this

8 paper, the factor decomposition model was used to analyze the influencing factors and effects of carbon dioxide

9 emissions. Causal chain model of elastic decoupling was established. The historical decoupling state between carbon dioxide emissions and economic growth and the decoupling effect of its influencing factors were analyzed. The prediction model of carbon dioxide emissions was used to explore the change trend of China's carbon dioxide emissions and its peak in the short and medium term in the future. The elastic decoupling trend between carbon dioxide emissions and economic growth was predicted. The results show that economic growth is the main force driving carbon dioxide emissions. Both energy intensity and energy consumption structure have a strong inhibiting effect on carbon dioxide emissions except for a few years, but the former has a more significant inhibiting effect than the latter. In general, the elastic decoupling between carbon dioxide emissions and economic growth has experienced a state from weak decoupling to growth linkage and then to weak decoupling. And this weak decoupling trend will continue to increase in the short and medium term. During the 14th Five-year and 15th Five-year period, if the average annual economic growth rate will be maintained at $4.61 \%$ to $5.85 \%$, and energy intensity will be reduced by $16.14 \%$ to $18.37 \%$, and the proportion of non-fossil energy in the energy consumption structure at the end of the $14^{\text {th }}, 15^{\text {th }}$ and $16^{\text {th }}$ Five-Year Plan period will be around $19.9 \%$, $23.2 \%$ and $26.1 \%$, respectively, then the intensity of carbon dioxide emissions will continue to decline. It is expected to reach the peak of carbon dioxide emissions between 10,453 and 10,690 billion tons from 2025 to 2027. And the earlier the peak time is, the smaller the peak is, which would provide valuable time for carbon neutrality and room to reduce carbon dioxide emissions in the medium and long term. 


\section{Introduction}

Peaking carbon dioxide emissions is not only one of China's international commitments in global climate

negotiations, but also an inevitable choice for China to achieve structural transformation and high-quality

development. China has made important contributions to adopting the Paris Agreement and has made active

efforts toward implementing it. In November 2014, the Chinese government announced that China plans to peak

carbon dioxide emissions around 2030 and will strive to reach the peak as soon as possible in the China-US Joint

Statement on Climate Change. In September 2020, the Chinese government announced at Climate Ambition

Summit that China would scale up its nationally determined contributions and adopt more vigorous policies and

measures. We aim to peak carbon dioxide emissions before 2030 and achieve carbon neutrality before 2060 .

China will lower its carbon dioxide emissions per unit of GDP by over 65 percent from the 2005 level, increase

the share of non-fossil fuels in primary energy consumption to around 25 percent, and so on. In December 2020,

the Central Economic Work Conference further pointed out that " China will seize time to formulate an action plan for peaking carbon dioxide emissions before 2030. The country will support areas with favorable conditions

Economic Work Conference. China will still adjust and optimize industrial structure and energy structure, promote coal consumption to peak as soon as possible, vigorously develop new energy, and improve the dual control system of energy consumption." Peaking carbon dioxide emissions is an important turning point in the transformation of economic development mode and an important node in the eventual realization of carbon neutrality. The earlier the peak time, the more conducive to achieving the goal of carbon neutrality. Therefore, in the "14th Five-Year Plan" and beyond in the medium and long term, our country must attach great importance to adjusting industrial structure and strengthening technological progress to save energy and improve efficiency. It is important to encourage green, low-carbon ways of life and production, and seek development opportunities and impetus from green development. Peaking carbon emissions before 2030 will force a green and low-carbon 
transformation of the energy structure. Therefore, exploring the path of carbon emissions peaking and the further decoupling between it and economic growth is of great significance to achieving high-quality development.

There are many methods to explore the influencing factors of carbon emissions, such as structural decomposition method, "IPAT" model, STIRPAT model, Granger causality test and so on. The IPAT equation means that the environment will be affected by the population, wealth, and technological level (Ehrlich and Holdren, 1971). Haroldo et al. (2019) analyzed the impact of population density on urban carbon dioxide emissions. However, this model has certain limitations and can only analyze the proportional changes in the environment and its influencing factors. Therefore, scholars have expanded the model to explore the relationship between carbon emissions and its influencing factors (Ang and Zhang, 1998). In addition, many scholars have added other economic and social factors to analyze this model (Lin et al., 2016). Based on the analysis of the majority of scholars, indicators such as the level of urbanization, industrial structure, and energy utilization will all have a significant impact on carbon emissions (Wang et al., 2019). Du et al. (2018) studied the driving factors of energy-related $\mathrm{CO}_{2}$ emissions changes in China's energy-intensive industries based on the logarithmic mean index (LMDI) method. Quan et al. (2020) also used the LMDI decomposition model to decompose the factors affecting the carbon emissions of China's logistics industry from five aspects: carbon emission coefficient, population size, and energy structure, and concluded that energy structure is the main limiting factor affecting carbon emissions in the logistics industry. Jiang and Han (2019) compared the carbon intensity of different countries based on the level of economic development and the perspective of industrial transfer. Wang and He (2020) used the LMDI method to decompose the influencing factors of carbon dioxide emissions in China's provinces and found that different influencing factors have different effects on carbon emissions in different regions. 
72 that can be reached in the future, and the time interval during which changes in carbon emissions will reverse.

73 Domestic and foreign scholars' prediction methods for peak carbon emissions can be roughly classified into three

74 categories. One is to judge whether there is an inflection point through the EKC model. If there is an inflection

75 point, it means that there is a peak in carbon emissions (Lin and Jiang, 2009). The other is to first decompose the influencing factors of carbon emissions. Among them, the models that can be used include STIRPAT model,

IPAT model and logarithmic average Di decomposition model, etc., and then combined with scenario analysis

to predict the future carbon emission trend (Liu et al., 2018). Part of the research will also directly use scenario analysis to predict future carbon emissions (Guan et al., 2008; Liu et al., 2018; Li, 2019; Zhang, 2020). Tian et al. (2019) analyzed the carbon footprint of China's industrial supply chain based on a life cycle assessment model of input and output. Huang et al. (2019), Li et al. (2018), and Zhang (2017) related to the third prediction method that constructs a system model to directly predict carbon emissions. Such methods include gray prediction models and CGE models. Lu et al. (2020) used the back propagation neural network (BP) model optimized by the particle swarm optimization (PSO) algorithm to predict the future carbon emissions of the heavy chemical industry. The results showed that the energy processing industry, the steel industry and the building materials industry during the forecast period. The proportion of medium carbon emissions accounts for a larger proportion of the carbon emissions of the heavy chemical industry. Li et al. (2021) established a prediction model through system dynamics in order to accurately predict the peak of carbon emissions from the provincial construction industry, and obtained a more reasonable low-carbon development route map. established the Tapio decoupling indicator system to analyze the decoupling between the economic growth of the European transportation industry from 1970 to 2001 . According to the decoupling value, the state of the 
decoupling elasticity is divided into connection, decoupling, and negative decoupling. Domestic scholars' research on decoupling theory mainly focuses on energy and environment. Qi et al. (2015) applied it at the provincial level. Guo et al. (2017) applied it at the regional level. Ma (2016) applied it at aspect of life. Zhao (2006) used a relative "decoupling" and "recoupling" theories and concluded that China's economy and energy have been in a weak decoupling relationship since the early 1980s, and there has been an expansionary recoupling trend in recent years. Liu (2014) used the elastic decoupling method to analyze the weak decoupling of carbon emissions and economic growth in Henan Province from 2000 to 2010. Yu et al. (2020) used the Tapio decoupling model to examine the relationship between the economic growth of low-carbon pilot cities. It is proposed that for low-carbon mature cities, vigorously developing renewable energy and increasing R\&D investment are effective ways to reduce emissions. Jie et al. (2020) used a system dynamics model to assess the potential of carbon decoupling in China's energy mining industry. From 2020 to 2030, it is estimated that China's coal, oil, and natural gas extraction industry will show a weak decoupling effect under the baseline scenario from carbon emissions and GDP growth. And there will be a strong decoupling effect from 2024 to 2030 under the planning scenario.

In summary, the existing literature has conducted research on peaking carbon emissions and its decoupling from economic growth, which provides a useful reference for the study of this article. Few documents have studied and judged the issue of peaking carbon emissions from the historical evolution of economic growth, energy intensity, and energy consumption structure. This article intends to analyze the influencing factors and effects of China's carbon dioxide emissions, and predict economic growth, energy intensity and primary energy consumption structure in scenarios. It intends to construct a carbon dioxide emissions prediction model from the perspective of economic growth, energy intensity and energy consumption structure, and combine it with scenario analysis. The change trend of China's carbon dioxide emissions in the medium and long term in the future will be predicted. The peak of carbon emissions will be researched and judged. The elastic decoupling 
117 between carbon emissions and economic growth will be predicted. It provides scientific reference for the

118 formulation of action plan for peaking carbon emissions before 2030.

\section{Carbon emission prediction model construction}

120

122

123

124

125

\subsection{Factor decomposition model of carbon emissions}

In this paper, the Logarithmic Mean Divisia Index (LMDI) of carbon emissions was constructed from the perspectives of population size, economic development level, energy intensity, and carbon intensity of energy consumption:

$$
C=\sum_{j=1}^{n} P \times \frac{Y}{P} \times \frac{E}{Y} \times \frac{E_{j}}{E} \times \frac{C_{j}}{E_{j}}=\sum_{j=1}^{n} P \times Y P \times I \times S E_{j} \times F_{j}
$$

Where, $j$ represents the type of energy. $P, Y, E$, and $C$ represent the population, GDP, energy consumption and carbon dioxide emissions, respectively. Then, $Y P=Y / P$ represents per capita GDP. $I=E / Y$ represents

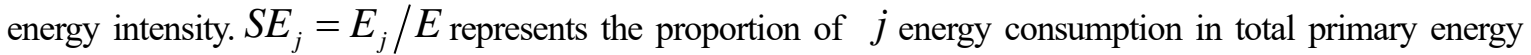
consumption. $F_{j}$ is the carbon emission coefficient of the $j$-th primary energy. Unless there is a major technological change, $F_{j}$ is a constant. Therefore, the carbon emissions coefficient per unit energy is determined by the energy consumption structure.

This model can be used to determine the carbon increase factors and carbon reduction factors. The comprehensive effect of carbon emissions from energy consumption is:

$$
\Delta C=\Delta C_{P}+\Delta C_{Y P}+\Delta C_{I}+\Delta C_{S E}+\Delta C_{F}
$$

In the above formula, the effect value of each decomposition factor is expressed as:

$$
\Delta C_{P}=\sum_{j=1}^{n} \omega \cdot \ln \frac{P^{t}}{P^{0}}
$$




$$
\Delta C_{Y P}=\sum_{j=1}^{n} \omega \cdot \ln \frac{Y P^{t}}{Y P^{0}}
$$

137

$$
\Delta C_{I}=\sum_{j=1}^{n} \omega \cdot \ln \frac{I^{t}}{I^{0}}
$$

138

$$
\Delta C_{S E}=\sum_{j=1}^{n} \omega \cdot \ln \frac{S E_{j}^{t}}{S E_{j}^{0}}
$$

139

$$
\Delta C_{F}=\sum_{j=1}^{n} \omega \cdot \ln \frac{F_{j}^{t}}{F_{j}^{0}}
$$

Among them, $t$ represents the analysis period, 0 represents the base period, and the common weight in the above formula is:

$$
\omega=\left(C_{j}^{t}-C_{j}^{0}\right) /\left(\ln C_{j}^{t}-\ln C_{j}^{0}\right)
$$

Since the primary energy consumption carbon emission coefficient is constant during the sample period, that is $F_{j}^{0}=F_{j}^{t}$, therefore, $\Delta C_{F}=0$. The comprehensive effect of energy consumption on carbon emissions 145 is further expressed as:

$$
\Delta C=\Delta C_{P}+\Delta C_{Y P}+\Delta C_{I}+\Delta C_{S E}
$$

the influencing factors of carbon emissions was decomposed and analyzed.

\subsection{Elastic decoupling model of carbon emissions} economic growth:

152

$$
e_{(C, Y)}=\frac{\Delta C}{C} / \frac{\Delta Y}{Y}=\left(\frac{\Delta E}{E} / \frac{\Delta Y}{Y}\right) \times\left(\frac{\Delta C}{C} / \frac{\Delta E}{E}\right)=e_{(E, Y)} \times e_{(C, E)}
$$


Among them, $e_{(C, Y)}$ is the ratio of the growth rate of carbon emissions to the growth rate of GDP, which represents the elastic decoupling index between carbon emissions and economic growth; $e_{(E, Y)}$ is the ratio of energy consumption growth rate to GDP growth rate, which represents the elastic decoupling index between energy consumption and economic growth; $e_{(C, E)}$ is the ratio of the growth rate of carbon emissions to the growth rate of energy consumption, and represents the elastic decoupling index between carbon emissions and energy consumption.

The effect of decoupling index of carbon emissions based on LMDI decomposition method is expressed as:

$$
\Delta e_{(C, Y)}=\Delta e_{(C, Y)}^{E}+\Delta e_{(C, Y)}^{Y}
$$

Among them, $\Delta e_{(C, Y)}$ represents the change of the carbon emission elasticity index, $\Delta e_{(C, Y)}^{E Y}=\frac{e_{(C, Y)}^{t}-e_{(C, Y)}^{0}}{\ln e_{(C, Y)}^{t}-\ln e_{(C, Y)}^{0}} \times \ln \frac{e_{(E, Y)}^{t}}{e_{(E, Y)}^{0}}$ indicates the impact of the elastic decoupling between energy consumption and economic growth on the decoupling of carbon emissions, $\Delta e_{(C, Y)}^{C E}=\frac{e_{(C, Y)}^{t}-e_{(C, Y)}^{0}}{\ln e_{(C, Y)}^{t}-\ln e_{(C, Y)}^{0}} \times \ln \frac{e_{(C, E)}^{t}}{e_{(C, E)}^{0}}$ indicates the impact of the elastic decoupling of carbon dioxide emissions from energy consumption on the decoupling of carbon emissions.

\subsection{Prediction model of carbon dioxide emissions}

Due to the complicated change trend of population size, population factors are not considered in the built model for the time being, and a carbon emission prediction model is constructed:

$$
C^{t}=Y^{t} \times \frac{E^{t}}{Y^{t}} \times\left(\sum_{j=1}^{n} \frac{E_{j}^{t}}{E^{t}} \times \frac{C_{j}^{t}}{E_{j}^{t}}\right)=Y^{0}\left(1+r_{Y}\right)^{t} \times I_{0}\left(1-r_{I}\right)^{t} \times\left(\sum_{j=1}^{n} S E_{j} \times F_{j}\right)
$$

Among them, 0 represents the base period, and $t$ is the final period period. $[0, t]$ represents the time interval for analysis. $Y^{0}$ and $Y^{t}$ are the base period and final GDP respectively, $r_{Y}$ is the average annual growth rate of GDP during the analysis period. $I_{0}=E_{0} / Y_{0}$ is the base period energy intensity, $r_{I}$ it is the average annual 
173 rate of decline in energy intensity during the analysis period. The symbols of other parameters are the same as

174 before

\section{3. Data source and parameter determination}

176

\subsection{Source of data}

The data used are from the "China Energy Statistical Yearbook", "China Statistical Yearbook" and "New China Sixty Years Collection" over the years, and the sample period is 2000-2019. Since my country first proposed in 2009 that my country's carbon dioxide intensity per unit of GDP in 2020 will be $45 \%$ lower than that in 2005, the GDP will be at a constant price in 2005, and the national carbon dioxide emissions will be calculated using the carbon emission coefficient method. The carbon emission coefficients of energy coal, oil, and natural gas are $0.7476 \mathrm{~kg}$ carbon $/ \mathrm{kg}$ standard coal, $0.5825 \mathrm{~kg}$ carbon $/ \mathrm{kg}$ standard coal, and $0.4435 \mathrm{~kg}$ carbon/kg standard coal, respectively, using the recommended values of the National Development and Reform Commission.

\subsection{Parameter determination}

The parameters in the carbon emission prediction model are determined below. The GDP in 2019 was $60,671.593$ billion yuan (constant prices in 2005), and the energy intensity in 2019 was 0.8027 tons of standard coal per 10,000 yuan

\subsubsection{Gross Domestic Product (GDP)}

From the new normal stage to the current period of high-quality development, my country's GDP growth rate has slowed down, hovering at around 7\%. The quadratic parabolic model is used to predict GDP (as shown in Table 1). Based on the four scenarios of 2000-2019, 2005-2019, 2010-2019, and 2015-2019, the forecast results of GDP from 20202035 (constant prices in 2005) are shown in Table 1.

Table 1. Forecast of GDP from 2020 to 2035

Unit: 100 million yuan

\begin{tabular}{c|c|c|c|c}
\hline years & The first scenario & The second scenario & The third scenario & The fourth scenario \\
\hline 2020 & 650238.1 & 641235.7 & 643647.8 & 644326.6 \\
\hline 2021 & 690506.8 & 677778.8 & 681678.3 & 682532.6 \\
\hline 2022 & 732027.2 & 715113.4 & 720769.9 & 721742.6 \\
\hline
\end{tabular}




\begin{tabular}{|c|c|c|c|c|}
\hline 2023 & 774799.3 & 753239.8 & 760922.6 & 761956.6 \\
\hline 2024 & 818823.0 & 792157.8 & 802136.3 & 803174.7 \\
\hline 2025 & 864098.4 & 831867.5 & 844411.1 & 845396.7 \\
\hline 2026 & 910625.5 & 872368.8 & 887747.0 & 888622.8 \\
\hline 2027 & 958404.2 & 913661.8 & 932144.0 & 932852.9 \\
\hline 2028 & 1007434.6 & 955746.5 & 977602.0 & 978087.1 \\
\hline 2029 & 1057716.7 & 998622.8 & 1024121.1 & 1024325.2 \\
\hline 2030 & 1109250.5 & 1042290.8 & 1071701.3 & 1071567.4 \\
\hline 2031 & 1162035.9 & 1086750.4 & 1120342.5 & 1119813.6 \\
\hline 2032 & 1216073.0 & 1132001.7 & 1170044.8 & 1169063.8 \\
\hline 2033 & 1271361.8 & 1178044.7 & 1220808.2 & 1219318.0 \\
\hline 2034 & 1327902.3 & 1224879.3 & 1272632.7 & 1270576.3 \\
\hline 2035 & 1385694.4 & 1272505.6 & 1325518.2 & 1322838.5 \\
\hline "Fourteenth Five-Year" & & & & \\
\hline Average annual growth rate & 0.0585 & 0.0534 & 0.0558 & 0.0558 \\
\hline "Fifteenth Five-Year" & & & & \\
\hline Average annual growth rate & 0.0512 & 0.0461 & 0.0488 & 0.0486 \\
\hline "Sixteenth Five-Year" & & & & \\
\hline Average annual growth rate & 0.0455 & 0.0407 & 0.0434 & 0.0430 \\
\hline
\end{tabular}

3.2.2 Energy intensity obtained that in the "fourteenth five-year", "fifteenth five-year", and "sixteenth five-year" energy intensity scenarios. The reduction rate of energy intensity is $12.75 \%, 18.20 \%, 18.37 \%$, and $16.14 \%$, respectively.

\begin{tabular}{lcccc}
\hline years & The first scenario & The second scenario & The third scenario & The fourth scenario \\
\hline 2020 & 0.8273 & 0.7618 & 0.7611 & 0.7705 \\
2021 & 0.8050 & 0.7318 & 0.7309 & 0.7438 \\
2022 & 0.7834 & 0.7030 & 0.7018 & 0.7181 \\
2023 & 0.7623 & 0.6753 & 0.6739 & 0.6933 \\
2024 & 0.7418 & 0.6487 & 0.6470 & 0.6693 \\
2025 & 0.7218 & 0.6231 & 0.6213 & 0.6461
\end{tabular}




$\begin{array}{lllll}2026 & 0.7024 & 0.5986 & 0.5966 & 0.6238 \\ 2027 & 0.6835 & 0.5750 & 0.5728 & 0.6022 \\ 2028 & 0.6651 & 0.5524 & 0.5501 & 0.5814 \\ 2029 & 0.6472 & 0.5306 & 0.5282 & 0.5613 \\ 2030 & 0.6298 & 0.5097 & 0.5072 & 0.5419 \\ 2031 & 0.6129 & 0.4896 & 0.4870 & 0.5231 \\ 2032 & 0.5964 & 0.4704 & 0.4676 & 0.5050 \\ 2033 & 0.5803 & 0.4518 & 0.4490 & 0.4876 \\ 2034 & 0.5647 & 0.4340 & 0.4311 & 0.4707 \\ 2035 & 0.5495 & 0.4169 & 0.4140 & 0.4544\end{array}$

A Markov forecast model of primary energy consumption structure is constructed:

202

$$
\left(S E_{1}^{t}, S E_{2}^{t}, S E_{3}^{t}, S E_{4}^{t}\right)=\left(S E_{1}^{0}, S E_{2}^{0}, S E_{3}^{0}, S E_{4}^{0}\right) P^{t}
$$

Among them, $S E_{1}^{t}, S E_{2}^{t}, S E_{3}^{t}, S E_{4}^{t}$ are the predicted values of the proportion of coal, oil, natural gas and 204

non-fossil energy in the total primary energy consumption during the reporting period, respectively. $S E_{1}^{0}, S E_{2}^{0}$, $S E_{3}^{0}, S E_{4}^{0}$ are the proportions of coal, oil, natural gas, and non-fossil energy in the total primary energy 206 consumption in the base period respectively. The primary energy consumption structure in the base period in 207 2019 is $\left(S E_{1}^{0}, S E_{2}^{0}, S E_{3}^{0}, S E_{4}^{0}\right)=(57.7 \%, 18.9 \%, 8.1 \%, 15.3 \%) . \mathrm{P}$ is the average one-step transition 208

probability matrix per year during the analysis period. And its determination is also calculated from the geometric average of the one-step transition probability matrix year by year during the analysis period. That is, $P^{t}=P_{1} \times P_{2} \times \cdots \times P_{t}$, where $P_{i}$ is the one-step transition probability matrix from the i-1year to the i year. predicted as shown in Table 3.

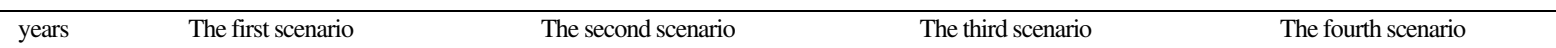




\begin{tabular}{|c|c|c|c|c|c|c|c|c|c|c|c|c|c|c|c|c|}
\hline & Coal & oil & $\begin{array}{c}\text { natural } \\
\text { gas }\end{array}$ & $\begin{array}{c}\text { non- } \\
\text { fossil } \\
\text { energy }\end{array}$ & Coal & oil & $\begin{array}{c}\text { natural } \\
\text { gas }\end{array}$ & $\begin{array}{l}\text { non- } \\
\text { fossil } \\
\text { energy }\end{array}$ & Coal & oil & $\begin{array}{c}\text { natural } \\
\text { gas }\end{array}$ & $\begin{array}{c}\text { non- } \\
\text { fossil } \\
\text { energy }\end{array}$ & Coal & oil & $\begin{array}{c}\text { natural } \\
\text { gas }\end{array}$ & $\begin{array}{c}\text { non- } \\
\text { fossil } \\
\text { energy }\end{array}$ \\
\hline 2020 & 57.2 & 18.7 & 8.4 & 15.7 & 56.8 & 18.9 & 8.5 & 15.8 & 56.6 & 19.0 & 8.5 & 15.9 & 56.2 & 19.0 & 8.7 & 16.1 \\
\hline 2021 & 56.6 & 18.6 & 8.7 & 16.1 & 55.9 & 19.0 & 8.9 & 16.2 & 55.4 & 19.2 & 9.0 & 16.4 & 54.7 & 19.1 & 9.2 & 16.9 \\
\hline 2022 & 56.1 & 18.4 & 9.0 & 16.4 & 55.0 & 19.0 & 9.3 & 16.7 & 54.3 & 19.3 & 9.4 & 16.9 & 53.3 & 19.2 & 9.7 & 17.7 \\
\hline 2023 & 55.6 & 18.3 & 9.3 & 16.8 & 54.1 & 19.1 & 9.7 & 17.1 & 53.3 & 19.4 & 9.9 & 17.4 & 52.0 & 19.4 & 10.3 & 18.4 \\
\hline 2024 & 55.1 & 18.2 & 9.5 & 17.2 & 53.3 & 19.1 & 10.0 & 17.5 & 52.3 & 19.6 & 10.3 & 17.9 & 50.6 & 19.5 & 10.8 & 19.2 \\
\hline 2025 & 54.6 & 18.0 & 9.8 & 17.5 & 52.5 & 19.1 & 10.4 & 18.0 & 51.2 & 19.7 & 10.7 & 18.3 & 49.3 & 19.6 & 11.3 & 19.9 \\
\hline 2026 & 54.2 & 17.9 & 10.1 & 17.9 & 51.7 & 19.2 & 10.8 & 18.4 & 50.3 & 19.8 & 11.1 & 18.8 & 48.0 & 19.7 & 11.8 & 20.6 \\
\hline 2027 & 53.7 & 17.7 & 10.4 & 18.2 & 50.9 & 19.2 & 11.2 & 18.8 & 49.3 & 19.9 & 11.6 & 19.2 & 46.8 & 19.8 & 12.2 & 21.2 \\
\hline 2028 & 53.2 & 17.6 & 10.7 & 18.5 & 50.1 & 19.2 & 11.6 & 19.1 & 48.4 & 20.0 & 12.0 & 19.6 & 45.6 & 19.9 & 12.7 & 21.9 \\
\hline 2029 & 52.8 & 17.4 & 10.9 & 18.9 & 49.3 & 19.2 & 11.9 & 19.5 & 47.5 & 20.1 & 12.4 & 20.0 & 44.4 & 19.9 & 13.1 & 22.5 \\
\hline 2030 & 52.3 & 17.3 & 11.2 & 19.2 & 48.6 & 19.2 & 12.3 & 19.9 & 46.6 & 20.2 & 12.8 & 20.4 & 43.2 & 20.0 & 13.6 & 23.2 \\
\hline 2031 & 51.9 & 17.2 & 11.5 & 19.5 & 47.8 & 19.3 & 12.7 & 20.2 & 45.7 & 20.3 & 13.2 & 20.7 & 42.1 & 20.1 & 14.0 & 23.8 \\
\hline 2032 & 51.4 & 17.0 & 11.8 & 19.8 & 47.1 & 19.3 & 13.0 & 20.6 & 44.9 & 20.4 & 13.6 & 21.1 & 41.0 & 20.2 & 14.4 & 24.4 \\
\hline 2033 & 51.0 & 16.9 & 12.0 & 20.1 & 46.4 & 19.3 & 13.4 & 20.9 & 44.1 & 20.5 & 14.0 & 21.4 & 40.0 & 20.3 & 14.8 & 25.0 \\
\hline 2034 & 50.6 & 16.7 & 12.3 & 20.4 & 45.7 & 19.3 & 13.8 & 21.2 & 43.3 & 20.6 & 14.4 & 21.7 & 38.9 & 20.4 & 15.2 & 25.5 \\
\hline 2035 & 50.2 & 16.6 & 12.6 & 20.7 & 45.0 & 19.3 & 14.1 & 21.5 & 42.5 & 20.7 & 14.8 & 22.0 & 37.9 & 20.5 & 15.6 & 26.1 \\
\hline
\end{tabular}

217 overall downward trend by 2030. In the second scenario, the proportion of non-fossil energy will be $19.9 \%$, and

218 the proportion of coal will decline even more by 2030. In the third scenario, the proportion of non-fossil energy

219 will be $20.4 \%$, and the proportion of coal will reach $46.6 \%$ by 2030 . In the fourth scenario, the proportion of

220 non-fossil energy will be $23.2 \%$, and the proportion of coal will decline the most by 2030 .

\section{4. Empirical analysis and forecast of China's carbon dioxide emissions}

\subsection{The historical evolution trend of carbon dioxide emissions and its influencing factors}




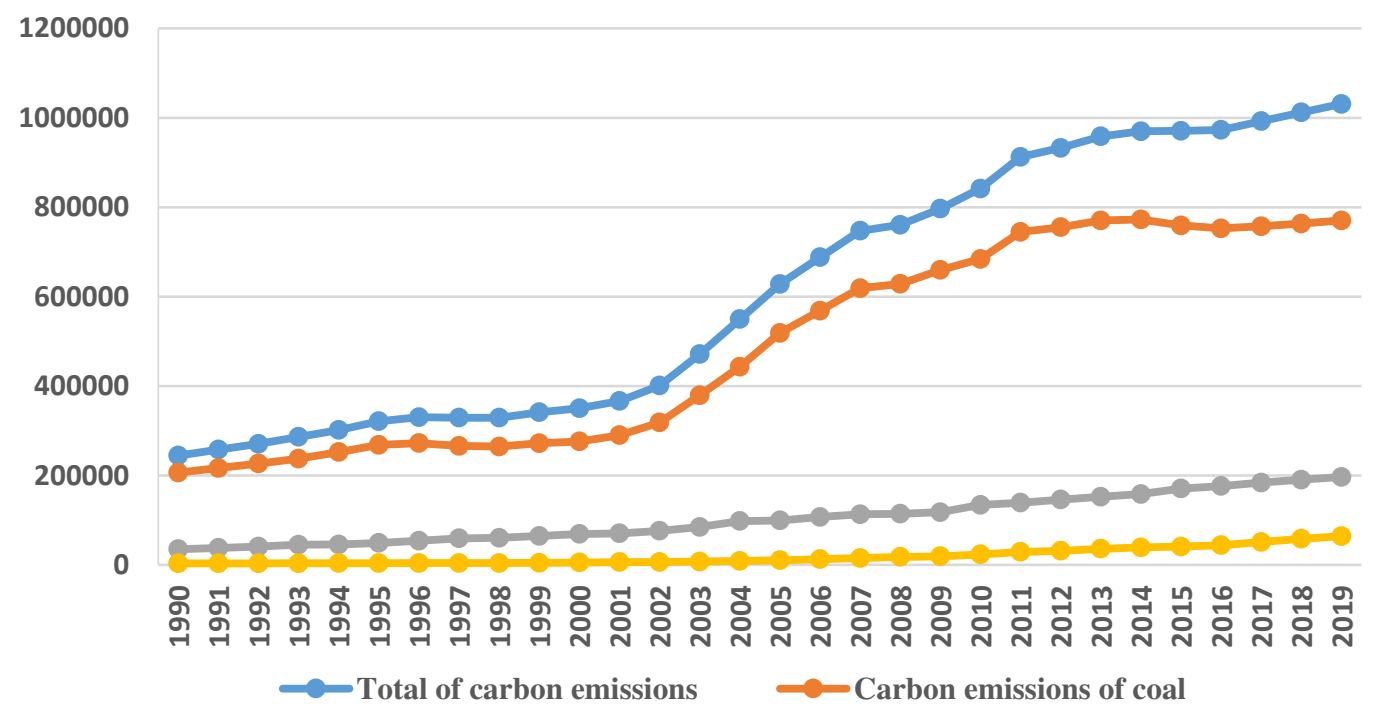

The trend of total carbon dioxide emissions is basically similar as the trend of carbon dioxide emissions from coal consumption, which is determined by China's resource endowments in Figure 1. emissions. However, China's economy entered the mid-term stage of rapid industrialization from 2003 to 2013

Due to the extensive economic development, coal consumption increased rapidly, which in turn led to a large amount of carbon dioxide emissions throughout the country. After 2013, China's economy has entered a new normal, and the growth rate of economy has slowed down, and industry structure faces transformation. So the growth rate of coal consumption slowed down, especially the growth rate of carbon dioxide emissions from coal consumption were very slowly in 2014 and 2015. There was a slight increase during 2016-2019, but it was basically controlled within 8 billion tons, indicating that coal has been effectively controlled after 2014, which has led to a slowdown in the growth of national carbon dioxide emissions. In recent years, the consumption of oil, natural gas and non-fossil energy has grown rapidly, replacing part of the consumption of coal and slowing down carbon dioxide emissions. On the whole, the total carbon dioxide emissions during the sample period have been increasing year by year. The growth rate of carbon dioxide emissions was relatively fast from 2000 to 2013 , 
242 and the growth rate of carbon dioxide emissions was slow from 2014 to 2019. At present, carbon emissions have

243 not reached a peak, but the growth rate has slowed down year by year. The carbon dioxide emissions from the consumption of oil and natural gas is increasing year by year.

246 population size, economic development level, energy intensity, and primary energy consumption structure on

247 carbon dioxide emissions can be obtained in Table 4. It can be seen that the population size and the level of economic development both promoted carbon dioxide emissions to varying degrees in the sample period. In

249 addition, energy intensity promoted carbon dioxide emissions during 2002-2004, and energy intensity have

250 different degrees of restraint on carbon dioxide emissions during other periods of time. During the individual

251 years of 2001, 2002, 2004 and 2010, China's primary energy consumption structure has a promoting effect on

252 carbon dioxide emissions. The energy consumption structure has a different degree of restraint and promotion

253 effect on carbon dioxide emissions during other years.

Table 4. The influence and contribution rate of various factors on carbon dioxide emissions

\begin{tabular}{|c|c|c|c|c|c|c|c|c|c|c|}
\hline \multirow[b]{2}{*}{ years } & \multicolumn{5}{|c|}{ Influence effect } & \multicolumn{5}{|c|}{ Contribution rate } \\
\hline & $\begin{array}{c}\text { Populatio } \\
\text { n size }\end{array}$ & $\begin{array}{l}\text { The level of } \\
\text { development }\end{array}$ & $\begin{array}{l}\text { Energy } \\
\text { intensity }\end{array}$ & $\begin{array}{l}\text { Energy } \\
\text { structure }\end{array}$ & $\begin{array}{l}\text { Total } \\
\text { effect }\end{array}$ & $\begin{array}{c}\text { Populatio } \\
\text { n size }\end{array}$ & $\begin{array}{l}\text { The level of } \\
\text { development }\end{array}$ & $\begin{array}{l}\text { Energy } \\
\text { intensity }\end{array}$ & $\begin{array}{l}\text { Energy } \\
\text { structure }\end{array}$ & $\begin{array}{l}\text { Total } \\
\text { effect }\end{array}$ \\
\hline 2000-2001 & 2602.5 & 26083.7 & -8349.8 & -4163.78 & 16172.7 & 16.1 & 161.3 & -51.6 & -25.75 & 100 \\
\hline 2001-2002 & 2568.6 & 30939.3 & -400.1 & 1268.77 & 34376.5 & 7.5 & 90.0 & -1.2 & 3.69 & 100 \\
\hline $2002-2003$ & 2709.8 & 38905.4 & 23780.8 & 5014.31 & 70410.2 & 3.8 & 55.3 & 33.8 & 7.12 & 100 \\
\hline 2003-2004 & 3025.6 & 46053.4 & 30225.4 & -910.82 & 78393.6 & 3.9 & 58.7 & 38.6 & -1.16 & 100 \\
\hline 2004-2005 & 3457.8 & 59986.0 & 11008.8 & 4211.05 & 78663.6 & 4.4 & 76.3 & 14.0 & 5.35 & 100 \\
\hline $2005-2006$ & 3672.8 & 75091.4 & -18453.6 & -417.10 & 59893.4 & 6.1 & 125.4 & -30.8 & -0.70 & 100 \\
\hline 2006-2007 & 3746.1 & 91688.7 & -35477.7 & -912.39 & 59044.7 & 6.3 & 155.3 & -60.1 & -1.55 & 100 \\
\hline $2007-2008$ & 3862.4 & 65584.9 & -47575.5 & -8630.61 & 13241.3 & 29.2 & 495.3 & -359.3 & -65.18 & 100 \\
\hline $2008-2009$ & 3871.5 & 66049.7 & -33136.8 & -669.57 & 36114.8 & 10.7 & 182.9 & -91.8 & -1.85 & 100 \\
\hline
\end{tabular}




\begin{tabular}{llllllllllll}
$2009-2010$ & 3954.3 & 78802.2 & -25101.7 & -12631.71 & 45023.1 & 8.8 & 175.0 & -55.8 & -28.06 & 100 \\
$2010-2011$ & 4200.1 & 75759.8 & -18044.4 & 9105.70 & 71021.2 & 5.9 & 106.7 & -25.4 & 12.82 & 100 \\
$2011-2012$ & 4494.7 & 65337.8 & -34537.8 & -15415.62 & 19879.1 & 22.6 & 328.7 & -173.7 & -77.55 & 100 \\
$2012-2013$ & 4667.5 & 66041.6 & -36597.2 & -8141.55 & 25970.3 & 18.0 & 254.3 & -140.9 & -31.35 & 100 \\
$2013-2014$ & 4881.5 & 64179.0 & -43004.2 & -14666.65 & 11389.6 & 42.9 & 563.5 & -377.6 & -128.77 & 100 \\
$2014-2015$ & 4930.0 & 61087.7 & -53015.8 & -12105.93 & 896.0 & 550.2 & 6818.1 & -5917.2 & -1351.2 & 100 \\
$2015-2016$ & 5262.0 & 59113.1 & -47995.6 & -14259.38 & 2120.2 & 248.2 & 2788.1 & -2263.8 & -672.56 & 100 \\
$2016-2017$ & 5493.3 & 60496.0 & -34595.0 & -11920.21 & 19474.1 & 28.2 & 310.6 & -177.6 & -61.21 & 100 \\
$2017-2018$ & 4568.6 & 60886.8 & -30674.9 & -15060.29 & 19720.3 & 23.2 & 308.8 & -155.5 & -76.37 & 100 \\
$2018-2019$ & 3649.6 & 56931.2 & -28461.9 & -13184.77 & 18934.2 & 19.3 & 300.7 & -150.3 & -69.63 & 100 \\
\hline
\end{tabular}

From the perspective of the contribution rate of various influencing factors to carbon dioxide emissions, the

256 positive contribution rate of economic development level to carbon dioxide emissions is the highest, followed

257 by population size. Energy intensity and energy structure have a negative effect on carbon dioxide except for a

258 few years. The negative contribution rate of energy intensity to carbon dioxide emissions is the highest, followed

259 by the primary energy consumption structure. From the overall changes in the contribution rate of various

260 influencing factors to carbon emissions, the contribution rate of the economic development level shows a

261 fluctuating upward trend, indicating that the main driving force for the increase in carbon dioxide emissions is

262 the increasing level of economic development. The contribution rate of population size to carbon dioxide

263 emissions fluctuates, but the overall change is not significant. The overall negative contribution rate of energy

264 intensity has shown an increasing trend, indicating that the increase in energy utilization can curb the increase in

265 carbon dioxide emissions. However, after 2016, the negative contribution rate of energy intensity to carbon

266 dioxide emissions has shown a downward trend year by year. The overall negative contribution rate of the

267 primary energy consumption structure is on the rise, indicating that the adjustment and optimization of China's

268 primary energy consumption structure have significantly inhibited carbon dioxide emissions. 


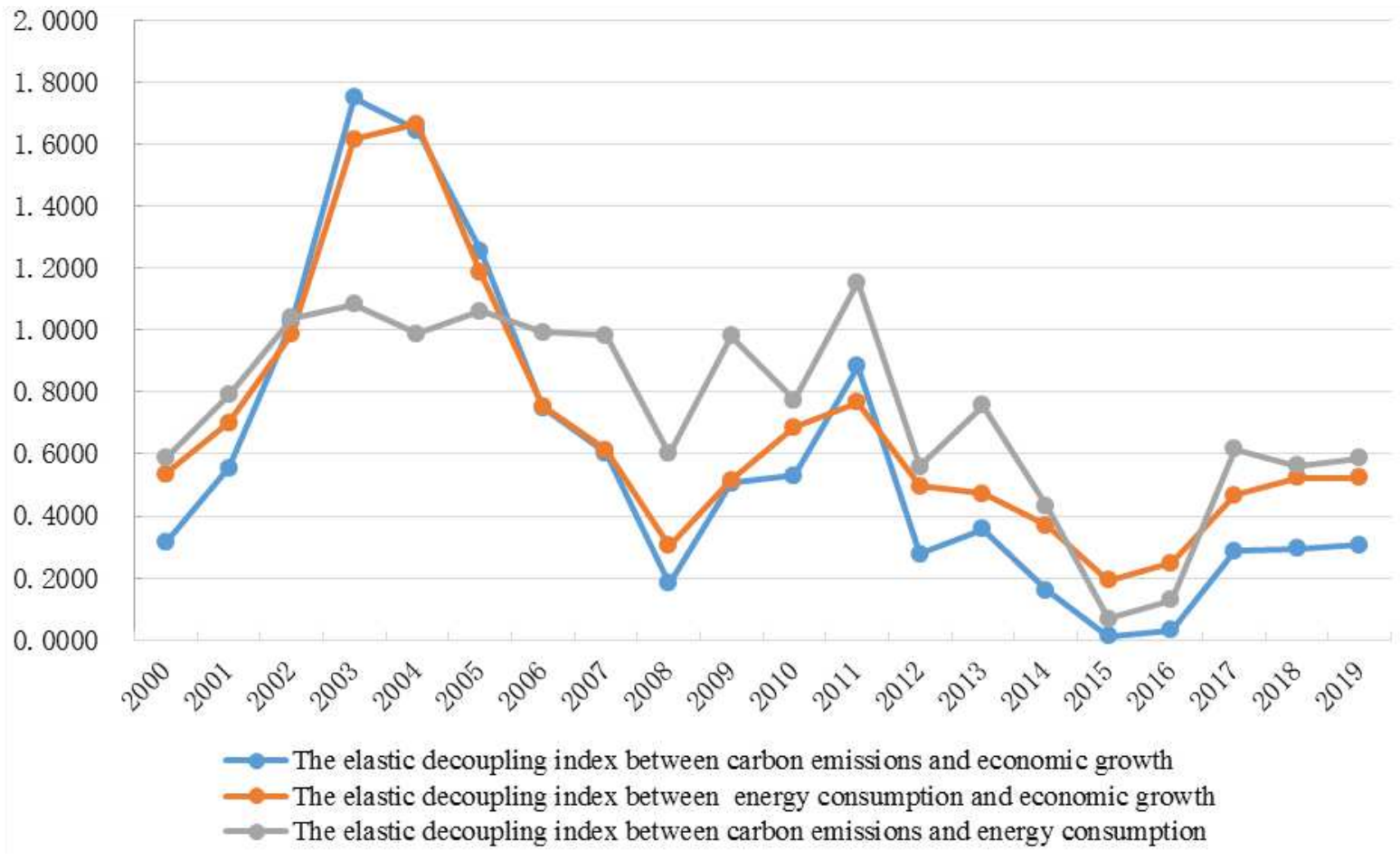

Figure 2. The elastic decoupling index between carbon emissions, energy consumption and economic growth from 2000 to 2019

In Figure 2, the elastic decoupling index between carbon dioxide emissions and economic growth has increased year by year from 2000 to 2003 . The elastic decoupling state between carbon dioxide emissions and economic growth had changed from weak decoupling state to growth link state. And the growth link state remained unchanged until 2005. After 2005, it dropped to a minimum quickly in 2008, and then rose to 0.8836 in 2011. The possible reason is that China's economic growth reached its peak from 2002 to 2007, and then economic growth gradually declined. However, the basic trend of the overall improvement of the economic growth has not changed. Compared with previous years, the economic environment has undergone major changes. Affected by the financial crisis around 2008, China's economic growth has been under pressure from a 
284 decline in growth since 2008. From 2007 to 2010, the elastic decoupling index of carbon emissions fluctuated

285 down and fluctuated up, but it has been weakly decoupled basically. The elastic decoupling index between carbon

286 dioxide emissions and economic growth was a weak decoupling state during 2015 - 2016. The decline in carbon

287 dioxide emissions is mainly due to the decline in energy intensity brought about by energy conservation and

288 technological progress. The industrial structure and energy consumption structure are basically stable. Energy

289 intensity and energy structure have little effect on the elastic decoupling state between carbon dioxide emissions

290 and economic growth.

From the causal chain model of the elastic decoupling index between carbon emissions and economic

292 growth, the elastic decoupling index between carbon dioxide emissions and economic growth is determined by

293 the elastic decoupling index between energy consumption and economic growth and the elastic decoupling index

294 between carbon dioxide emissions and energy consumption. Using LMDI decomposition method, the

295 decoupling effect of the above two indexes on the elastic decoupling index between carbon dioxide emissions

296 and economic growth and their contribution are shown in Table 5.

297 Table 5. The decomposition effect of the elastic decoupling index between carbon dioxide emissions and economic 298 growth and the contribution rate of each effect

\begin{tabular}{clllll}
\hline & \multicolumn{3}{c}{ Decoupling effect } & \multicolumn{2}{c}{ Decoupling contribution rate } \\
\cline { 2 - 6 } years & $\Delta e_{(C, Y)}^{E Y}$ & $\Delta e_{(C, Y)}^{C E}$ & $\Delta e_{(C, Y)}$ & $\Delta e_{(C, Y)}^{E Y} \%$ & $\Delta e_{(C, Y)}^{C E} \%$ \\
\hline $2000-2001$ & 0.1134 & 0.1260 & 0.2395 & 0.4736 & 0.5264 \\
$2001-2002$ & 0.2630 & 0.2102 & 0.4732 & 0.5559 & 0.4441 \\
$2002-2003$ & 0.6680 & 0.0549 & 0.7229 & 0.9241 & 0.0759 \\
$2003-3004$ & 0.0514 & -0.1565 & -0.1051 & -0.4887 & 1.4887 \\
$2004-2005$ & -0.4911 & 0.1022 & -0.3888 & 1.2629 & -0.2629 \\
$2005-2006$ & -0.4422 & -0.0645 & -0.5067 & 0.8728 & 0.1272 \\
$2006-2007$ & -0.1406 & -0.0059 & -0.1465 & 0.9599 & 0.0401 \\
$2007-2008$ & -0.2459 & -0.1734 & -0.4193 & 0.5865 & 0.4135 \\
$2008-2009$ & 0.1663 & 0.1553 & 0.3217 & 0.5171 & 0.4829 \\
$2009-2010$ & 0.1487 & -0.1225 & 0.0261 & 5.6894 & -4.6894
\end{tabular}




\begin{tabular}{llllll}
$2010-2011$ & 0.0767 & 0.2754 & 0.3522 & 0.2179 & 0.7821 \\
$2011-2012$ & -0.2275 & -0.3791 & -0.6066 & 0.3751 & 0.6249 \\
$2012-2013$ & -0.0149 & 0.0965 & 0.0816 & -0.1828 & 1.1828 \\
$2013-2014$ & -0.0612 & -0.1374 & -0.1986 & 0.3083 & 0.6917 \\
$2014-2015$ & -0.0385 & -0.1084 & -0.1469 & 0.2619 & 0.7381 \\
$2015-2016$ & 0.0055 & 0.0133 & 0.0188 & 0.2914 & 0.7086 \\
$2016-2017$ & 0.0737 & 0.1826 & 0.2562 & 0.2876 & 0.7124 \\
$2017-2018$ & 0.0329 & -0.0266 & 0.0063 & 5.2320 & -4.2320 \\
$2018-2019$ & -0.0002 & 0.0120 & 0.0118 & -0.0211 & 1.0211 \\
\hline
\end{tabular}
from 2000 to 2001. The increase in the elastic decoupling index between energy consumption and economic 301 growth increased the carbon emissions decoupling index by 0.1134 , and the increase in the elastic decoupling index between carbon dioxide emissions and energy consumption increased the carbon emissions decoupling index by 0.1260 . It shows that the increase of elastic decoupling index between energy consumption and economic growth and the increase of elastic decoupling index between carbon emissions and energy consumption jointly inhibit the further decoupling between carbon emissions and economic growth during 20002001. Similar to exploring the reasons for the change of carbon emission index in other years, the following results can be seen. Economic growth and energy consumption structure jointly suppressed the further decoupling between carbon emissions and economic growth during 2001-2003, 2008-2009, 2010-2011, and 2015-2017. Economic growth and energy consumption structure jointly promoted a further decoupling between carbon emissions and economic growth during 2005-2008 .

In some years, the above two indexes have inconsistent effects on the elastic decoupling between carbon emissions and economic growth. The increase in the elastic decoupling index between energy consumption and economic growth inhibited the elastic decoupling between carbon emissions and economic growth from 2003 to 2004 , but the decrease in the elastic decoupling index between carbon emissions and energy consumption promoted the decoupling between carbon emissions and economic growth. But the inhibition effect of the former 
317 index between energy consumption and economic growth promoted the elastic decoupling between carbon emissions and economic growth, and the increase in the elastic decoupling index between carbon emissions and energy consumption inhibited the elastic decoupling between carbon emissions and economic growth. But the former's promotion effect was greater than the latter's inhibitory effect. The increase of the elastic decoupling index between energy consumption and economic growth restrained the elastic decoupling index between carbon emissions and economic growth, while the decrease of the elastic decoupling index between carbon emissions and energy consumption promoted the elastic decoupling index between carbon emissions and economic growth. During 2009-2010 and 2017-2018, the increase of the elastic decoupling index between energy consumption and economic growth inhibited the elastic decoupling between carbon emissions and economic growth, and the decrease of the elastic decoupling index between carbon emissions and energy consumption promoted the elastic decoupling between carbon emissions and economic growth. But the inhibitory effect of the former is greater than the promotion of the latter. During 2012-2013 and 2018-2019, the decrease of the elastic decoupling index between energy consumption and economic growth promoted the elastic decoupling between carbon emissions and economic growth, but the increase in the elastic decoupling index between carbon emissions and energy consumption inhibited. However, the former's promotion effect is less than the latter's restraining effect.

\subsubsection{Carbon dioxide emission}

Bring the predicted values of the three parameters of GDP, energy intensity, and primary energy consumption structure into the above constructed carbon dioxide prediction model to predict the change trend of 
dioxide emissions under various scenarios are shown in Table 6.

\begin{tabular}{|c|c|c|c|c|}
\hline years & The first scenario & The second scenario & The third scenario & The fourth scenario \\
\hline 2020 & 1131860.5 & 1025490.0 & 1026795.5 & 1036430.6 \\
\hline 2021 & 1162512.1 & 1032660.8 & 1033973.8 & 1045432.1 \\
\hline 2022 & 1192056.2 & 1038128.8 & 1039713.9 & 1052855.9 \\
\hline 2023 & 1220479.1 & 1041996.3 & 1044087.1 & 1058778.0 \\
\hline 2024 & 1247766.3 & 1044363.4 & 1047161.8 & 1063279.2 \\
\hline 2025 & 1273910.5 & 1045326.1 & 1049009.4 & 1066438.8 \\
\hline 2026 & 1298909.3 & 1044972.5 & 1049694.6 & 1068330.6 \\
\hline 2027 & 1322758.7 & 1043389.3 & 1049285.2 & 1069025.4 \\
\hline 2028 & 1345463.9 & 1040661.4 & 1047843.6 & 1068598.5 \\
\hline 2029 & 1367030.3 & 1036868.4 & 1045430.1 & 1067115.6 \\
\hline 2030 & 1387464.2 & 1032081.7 & 1042106.8 & 1064642.6 \\
\hline 2031 & 1406772.7 & 1026375.8 & 1037930.1 & 1061244.1 \\
\hline 2032 & 1424972.6 & 1019819.3 & 1032953.5 & 1056982.0 \\
\hline 2033 & 1442073.0 & 1012477.5 & 1027234.1 & 1051912.4 \\
\hline 2034 & 1458089.9 & 1004411.6 & 1020818.4 & 1046094.3 \\
\hline 2035 & 1473038.8 & 995679.5 & 1013757.5 & 1039579.3 \\
\hline
\end{tabular}
will reach its peak in 2025 , with a peak value of 10,453 million tons. In the third scenario, carbon dioxide emissions will reach its peak in 2026, with a peak value of 10,453 million tons. In the fourth scenario, carbon

344 dioxide emissions will reach its peak in 2027 with a peak value of 10,690 million tons. The latter three scenarios can all achieve the goal of peaking carbon dioxide emissions before 2030 announced at Climate Ambition Summit. The result show that that the sooner the peak of carbon emissions is reached, the peak will be smaller, 
347 and the time left for carbon neutralization in 2060 will be longer. After peaking carbon dioxide emissions, which

348 will enter a plateau period of slow decline.

349 4.3.2 Prediction of carbon dioxide emissions intensity

350

Based on the predicted value of carbon dioxide emissions and the predicted value of GDP, the results of

351 carbon dioxide emissions intensity are shown in Table 7.

\begin{tabular}{|c|c|c|c|c|}
\hline years & The first scenario & The second scenario & The third scenario & The fourth scenario \\
\hline 2020 & 1.7407 & 1.5992 & 1.5953 & 1.6085 \\
\hline 2021 & 1.6836 & 1.5236 & 1.5168 & 1.5317 \\
\hline 2022 & 1.6284 & 1.4517 & 1.4425 & 1.4588 \\
\hline 2023 & 1.5752 & 1.3834 & 1.3721 & 1.3896 \\
\hline 2024 & 1.5239 & 1.3184 & 1.3055 & 1.3238 \\
\hline 2025 & 1.4743 & 1.2566 & 1.2423 & 1.2615 \\
\hline 2026 & 1.4264 & 1.1979 & 1.1824 & 1.2022 \\
\hline 2027 & 1.3802 & 1.1420 & 1.1257 & 1.1460 \\
\hline 2028 & 1.3355 & 1.0888 & 1.0719 & 1.0925 \\
\hline 2029 & 1.2924 & 1.0383 & 1.0208 & 1.0418 \\
\hline 2030 & 1.2508 & 0.9902 & 0.9724 & 0.9935 \\
\hline 2031 & 1.2106 & 0.9444 & 0.9264 & 0.9477 \\
\hline 2032 & 1.1718 & 0.9009 & 0.8828 & 0.9041 \\
\hline 2033 & 1.1343 & 0.8595 & 0.8414 & 0.8627 \\
\hline 2034 & 1.0980 & 0.8200 & 0.8021 & 0.8233 \\
\hline 2035 & 1.0630 & 0.7825 & 0.7648 & 0.7859 \\
\hline
\end{tabular}
2005 is $62.71 \%, 70.48 \%, 71.01 \%$, and $70.38 \%$, respectively. Except for the first scenario, the other three scenarios have achieved the goal of "By 2030, China's carbon dioxide emissions per unit of GDP will drop by more than $65 \%$ from 2005 " announced by the central government at the Climate Ambition Summit. 

decoupling effect are shown in Table 8.

\begin{tabular}{|c|c|c|c|c|}
\hline years & The first scenario & The second scenario & The third scenario & The fourth scenario \\
\hline 2020 & 0.4373 & 0.1227 & 0.1183 & 0.1465 \\
\hline 2021 & 0.4226 & 0.0961 & 0.0968 & 0.1236 \\
\hline 2022 & 0.4081 & 0.0699 & 0.0755 & 0.1010 \\
\hline 2023 & 0.3935 & 0.0440 & 0.0544 & 0.0786 \\
\hline 2024 & 0.3789 & 0.0184 & 0.0335 & 0.0565 \\
\hline 2025 & 0.3645 & -0.0069 & 0.0127 & 0.0347 \\
\hline 2026 & 0.3499 & -0.0320 & -0.0078 & 0.0131 \\
\hline 2027 & 0.3355 & -0.0568 & -0.0282 & -0.0082 \\
\hline 2028 & 0.3212 & -0.0812 & -0.0484 & -0.0294 \\
\hline 2029 & 0.3068 & -0.1056 & -0.0684 & -0.0502 \\
\hline 2030 & 0.2924 & -0.1296 & -0.0883 & -0.0709 \\
\hline 2031 & 0.2782 & -0.1534 & -0.1081 & -0.0913 \\
\hline 2032 & 0.2639 & -0.1770 & -0.1276 & -0.1116 \\
\hline 2033 & 0.2497 & -0.2004 & -0.1471 & -0.1316 \\
\hline 2034 & 0.2356 & -0.2236 & -0.1664 & -0.1514 \\
\hline 2035 & 0.2215 & -0.2466 & -0.1856 & -0.1710 \\
\hline
\end{tabular}
to the historical evolution trend from 2005 to 2019, there will be a weak decoupling from 2020 to 2024, and a strong decoupling from 2025 to 2035, indicating that a strong decoupling between carbon emissions and economic growth can be achieved by the end of the "14th Five-Year Plan" period. According to the historical 
evolution trend from 2010 to 2019 , the weak decoupling will be realized from 2020 to 2025 , and the strong decoupling will be realized from 2026 to 2035 , indicating that the strong decoupling between carbon emissions and economic growth can be achieved at the beginning of the "15th Five-Year Plan". According to the historical evolution trend from 2015 to 2019, the weak decoupling will be realized from 2020 to 2026, and the strong decoupling will be realized from 2027 to 2035 , indicating that the strong decoupling between carbon emissions and economic growth can be achieved by the middle of the "15th Five-Year Plan". In short, if economic development, energy intensity, and energy consumption structure can follow the trend of changes since 2005, there will be a strong decoupling between carbon emissions and economic growth before 2030, which coincides with the peak time of carbon emissions.

\section{Conclusions and policy implications}

\subsection{Main conclusions}

In this paper, the factor decomposition model is used to analyze the influencing factors of carbon dioxide emission and their influencing effects.

paper uses a factor decomposition model to analyze the influencing factors and effects of carbon dioxide emissions. Combining the factor decomposition method and the elastic decoupling model, a causal chain model of elastic decoupling is constructed, and the historical trend of the decoupling state between carbon dioxide emissions and economic growth and the decoupling effect of influencing factors are all analyzed. China's economic development level, energy intensity and primary energy consumption structure are all predicted. From the perspectives of economic growth, energy intensity and energy consumption structure,a carbon dioxide emission prediction model are established. The trends and peaks of China's carbon dioxide emissions in the medium and long term are explored. The trend of the elastic decoupling between carbon dioxide emissions and economic growth in the medium and long term. The conclusion is as follows: 
(1) From the perspective of the influencing factors and effects of carbon dioxide emissions, since the

"Fifteenth Five-Year Plan", population size and economic growth can promote carbon dioxide emissions.

Energy intensity and energy consumption structure have a strong inhibitory effect on carbon dioxide emissions except for a few years, but the inhibitory effect of energy intensity on carbon dioxide emissions is more obvious than that of energy consumption structure. Since the contribution rate of the population size to carbon dioxide emissions fluctuates but the overall change is small, the contribution rate of the economic development level shows a fluctuating upward trend, indicating that the main driving force for the increase of carbon dioxide emissions is the increasing level of economic development. Since the negative contribution rate of energy intensity is generally increasing, it shows that the increase in energy utilization can help curb the increase in carbon emissions. However, since 2016, the negative contribution rate of energy intensity to carbon dioxide emissions has shown a downward trend year by year. The overall negative contribution rate of the primary energy consumption structure is on the rise, indicating that the adjustment of China's primary energy consumption structure has become more and more obvious in restraining carbon dioxide emissions.

(2) From the perspective of the forecast of peak carbon dioxide emissions, the average annual growth rate of GDP during the period from the "14th Five-Year Plan" to the "16th Five-Year Plan" is maintained at 4.61\%$5.85 \%$, and the decline in energy intensity remained at $16.14 \%-18.37 \%$, and the proportion of non-fossil energy consumption in the energy consumption structure at the end of the 14th, 15th and 16th Five-Year Plan was maintained at $19.9 \%, 23.2 \%$ and $26.1 \%$. The carbon dioxide emission reduction efforts will continue to increase, and it is expected that carbon emissions will reach a peak between 2025-2027, with a peak value of between 10,453 and 10,690 billion tons, and the sooner the peak is reached, the smaller the peak, which can provide for the future realization of carbon neutrality. Valuable time and room for carbon emissions reduction. And after reaching the peak, it enters a plateau period of slow decline. Therefore, during the "14th Five-Year Plan" period, economic growth needs to maintain a reasonable range, energy intensity needs to achieve the constraint targets 
in the "14th Five-Year Plan" outline, and the energy consumption structure needs to be further optimized to achieve the "14th Five-Year Plan" outline. Restricting these targets can further promote the early peak of carbon emissions and reduce the peak.

(3) The elastic decoupling between carbon dioxide emissions and economic growth has generally experienced a state of weak decoupling to growth connection and then to weak decoupling. In particular, the weak decoupling is very good in 2015 and 2016. This is because China's economy is in a new stage of "structural adjustment and transformation" in the past two years. The economic growth rate is stable at about $7 \%$, the industrial structure and energy consumption structure are basically stable, and the decline in carbon dioxide emissions is mainly due to the decline in energy intensity brought by energy conservation and technological progress. The degree of elastic decoupling between energy consumption and economic growth and the degree of elastic decoupling between carbon dioxide emissions and energy consumption have time differences in the impact of the degree of elastic decoupling between carbon dioxide emissions and economic growth. If economic development, energy intensity, and energy consumption structure can follow the evolution trend since 2005 , the weak decoupling between carbon dioxide emissions and economic growth after the "14th Five-Year Plan" will continue to increase, and it will be reversed before the "15th Five-Year Plan". It is a strong decoupling state, which coincides with the peak time of carbon.

\subsection{Policy implications}

Peaking carbon emissions is an important turning point in the transformation of economic development mode and an important node in the eventual realization of carbon neutrality. Through the analysis of this article, the following policy recommendations are put forward:

(1) Energy intensity need be vigorously reduced, energy need be saved, and energy utilization efficiency need further improved. Although the negative effect of energy intensity on the decoupling of carbon emissions 
434 is gradually diminishing, it is still a factor hindering the decoupling of China's economic development from

435 carbon emissions. The improvement of energy-saving and emission-reduction technologies is the fundamental

436 way to solve carbon emissions. Increasing investment in science and technology, updating talent introduction

437 measures, and improving independent innovation capabilities are the key ways to decouple China's economic

438 development from carbon emissions.

(2) Energy consumption structure need be vigorously optimized, and the proportion of non-fossil energy need be increased. The essence of the carbon peak problem is the energy transition. The speed and intensity of the development of renewable energy power generation to promote low-carbon energy structure. End-energy energy conservation and re-electrification can promote the decoupling between carbon emissions and energy consumption, which in turn can promote the early realization of carbon peak. Therefore, during the "14th FiveYear Plan" period, energy growth should be promoted to shift from fossil energy to non-fossil energy, the proportion of non-fossil energy need to be continuously increase, and rely on technological innovation to achieve an increase in the proportion of non-fossil electricity through electrification, informatization and intelligence. The measure of optimizing the energy structure, vigorously developing renewable energy, increasing the proportion of new low-carbon energy sources, promoting the development and utilization of new energy sources such as nuclear energy and solar energy should be adopted. The coal-based energy structure to achieve a balance between carbon dioxide emissions and economic growth. Then, the goal of decoupling state between carbon dioxide emissions and economic growth can be achieved.

(3) The policy implementation mechanism is the fundamental guarantee for peaking carbon emissions. Maintaining policy determination is particularly important for peaking carbon emissions. The top-level design of science needs to be implemented. Governance policies need to be formulated at the source. Economic development methods need to be transformed. Energy structure need to be transformed and optimized through 
market adjustment. Green upgrades need to be done autonomously. The consistency and effectiveness of policy

457 implementation need to be ensured. On the one hand, China must actively formulate and implement corresponding policies and regulations. At the same time, China must also levy carbon taxes, improve the carbon emission trading market, raise the threshold of high-polluting and high-energy-consuming industries, improve the elimination mechanism, and improve efficiency. On the other hand, China need strictly control the consumption of fossil energy, step up research and development and the use of clean energy. The government should actively formulate appropriate preferential policies to guide enterprises to carry out green reforms and innovations. The target of peaking carbon emissions should be regarded as a long-term strategic task supplemented by government guidance, and market regulation as the main task, so as to gradually realize the substitution of green and clean energy for fossil energy. The government need to take full advantage of peaking carbon peak plateau, strive to promote the improvement and development of the modern industrial system, promote the further optimization of the energy consumption structure. Improving technologies such as carbon capture and carbon storage after carbon peaks will provide a beneficial guarantee for achieving carbon neutrality.

\section{$469 \quad$ Availability of data and materials}

470 Not applicable.

\section{References:}

B. W Ang, F. Q Zhang, K. Chio. Factorizing changes in energy and environmental indicators through decomposition [J]. Energy, 1998, 23 (6): 489-495.

Du G, Sun C, Ouyang X et al. A decomposition analysis of energy-related $\mathrm{CO}_{2}$ emissions in Chinese six highenergy intensive industries. Journal of Cleaner Production, 2018, 184: 1102-1112. 
Guo B N, Lin J, Liu T F. An Empirical Analysis of Decoupling Relations between Economic Growth and Carbon Dioxide An Empirical Analysis of Decoupling Relations between Economic Growth and Carbon Dioxide. Ecological Economy, 2017, 33 (04): 25-29.

Guan D, Hubacek K, Weber C L, et al. The drivers of Chinese $\mathrm{CO}_{2}$ emissions from 1980 to 2030. Global Environmental Change. 2008, 18 (04): 626-634.

Haroldo V. Ribeiro and Diego Rybski and Jürgen P. Kropp. Effects of changing population or density on urban carbon dioxide emissions. Nature Communications, 2019, 10(1) : 30-42.

Huang Y, Shen L, Liu H. Grey relational analysis, principal component analysis and forecasting of carbon emissions based on long short-term memory in China. Journal of Cleaner Production, 2019, 209: 415-423.

Jiang W B, Han M Y, Tang Z P, Liu W D. International comparison of carbon intensity from the perspective of economic development levels and industrial transfers. Resource Science, 2019, 41(10): 1814-1823.

Jie L, Wang Z, Yu R X. Scenario simulation of carbon decoupling effect of China's energy mining industry. China Population, Resources and Environment of China, 2020, 30 (07): 47-56.

Li X M, Zhang Q. Factors Affecting Carbon Emission from Energy Consumption in Tianjin. Arid zone research, 2019, 36 (04): 997-1004.

Lin B, Ahmad I. Analysis of energy related carbon dioxide emission and reduction potential in Pakistan. Journal of Cleaner Production, 2016, 143 (01): 278-287.

Liu D N and Xiao, B W. Can China achieve its carbon emission peaking? A scenario analysis based on STIRPAT and system dynamics model. Ecological Indicators, 2018, 93 : 647-657.

Liu D, Xiao B. Can China achieve its carbon emission peaking? a scenario analysis based on STIRPAT and system dynamics model. Ecological indicators, 2018, 93 (10): 647-657. 
Lin B Q, Jiang Z J. Prediction Kuznets Curve Prediction and Analysis of Influencing Factors of Carbon Dioxide in China. Managing the world, 2009 (04): 27-36.

Li W, An C, Lu C. The assessment framework of provincial carbon emission driving factors: An empirical analysis of Hebei Province. Science of The Total Environment, 2018, 637-638:91-103.

Liu Q T. An Empirical Study on Decoupling Relation Between Carbon Emissions and Economic Growth—A Case Study of Henan Province. Economic Longitude, 2014, 31 (06): 132-136.

Li D Z, Huang G Y, Zhu S Y, Chen L, Wang J B. How to peak carbon emissions of provincial construction industry? Scenario analysis of Jiangsu Province. Renewable and Sustainable Energy Reviews, 2021,144:1364-0321.

$\mathrm{Lu} \mathrm{C}, \mathrm{Li} \mathrm{W}$, Gao S B. Driving determinants and prospective prediction simulations on carbon emissions peak for China's heavy chemical industry. Journal of Cleaner Production, 2020, 251:0959-6526.

Ma X W, Ye Y, Shi X Q, Zou L L. Decoupling economic growth from $\mathrm{CO}_{2}$ emissions: A decomposition analysis of China's household energy consumption. Advances in Climate Change Research, 2016, 7(3): 192-200.

Qi S Z, Lin S, Wang B B. Impact of Economic Growth Pattern of the Six Provinces of Central China on Regional Carbon Emission: Based on the Tapio Model and Lag Instrumental Variable Analysis of Panel Data. China Population, Resources and Environment of China, 2015, 25 (05): 59-66.

Quan C G, Cheng X J, Yu S S, Ye X. Analysis on the influencing factors of carbon emission in China's logistics industry based on LMDI method. Science of The Total Environment, 2020,734: 1-8.

Tapio P. Towards a theory of decoupling: degrees of decoupling in the EU and the case of road traffic in Finland between 1970 and 2001. Transport Policy, 2005, 12 (02): 137 -151. 
Wang H, He J, Wang H L, He J K. China's pre- $2020 \mathrm{CO}_{2}$ emission reduction potential and its influence. Frontiers in energy, 2019, 13 (03): 571-578.

Wang Y, He Y F. Spatiotemporal dynamics and influencing factors of provincial carbon emissions in China. World Geographic Studies, 2020, 29 (03): 512-522.

Xu C, Chen T. Estimation to China's $\mathrm{CO}_{2}$ emissions intensity in 2030:based on IPAT model of FA. China Population, Resources and Environment of China, 2016, 26 (07): 62-69.

Yu X, Chen N, Li M Q. Research on carbon emission characteristics and reduction pathways of low-carbon pilot cities in China. China Population, Resources and Environment of China, 2020, 30 (07): 1-9. environmental impacts. Ecological Economics, 2003, (03): 351-365.

Zhao Y P, Sun Q H, Duan N. Responsive relationship between economic development and energy consumption in China - a practical research based on comparative de-link and re-link theory[J]. Management of

Zhang G X, Su Z X. Analysis of Influencing Factors and Scenario Prediction of Transportation Carbon Emissions in the Yellow River Basin. Management Review, 2020, 32 (12): 283-294. Of Science and Technology Of Tianjin, 2017. 


\section{Consent for publication}

540 Not Applicable.

541 Competing interests

542 The authors declare that they have no competing interests.

\section{$543 \quad$ Funding}

544 The research is supported by the National Natural Science Foundation of China under grants 71804089 and

54571771138 , Humanities and Social Sciences Youth Foundation of Ministry of Education of China under grants

546 18YJCZH034 and 19YJC790128, Jiangsu Post-doctoral Research Funding Plan grants 2018K195C, Natural

547 Science Foundation of Shandong Province in China grant ZR2018LG003.

548 Authors' contributions

549 Weifeng Gong: Conceptualization, Methodology, Writing - original, Funding acquisition.

550 Chuanhui Wang: Formal analysis, Writing - review \& editing.

551 Zhenyue Fan: Investigation, Project administration.

552 Yang Xu: Investigation, Formal analysis.

553 Acknowledgements

554 This paper benefited from years of thinking about these issues and the discussion with many colleagues related

555 to economics at that time. In particular, I would like to thank the chief editor of this journal and the anonymous reviewers for their helpful comments on future drafts. 


\section{Figures}

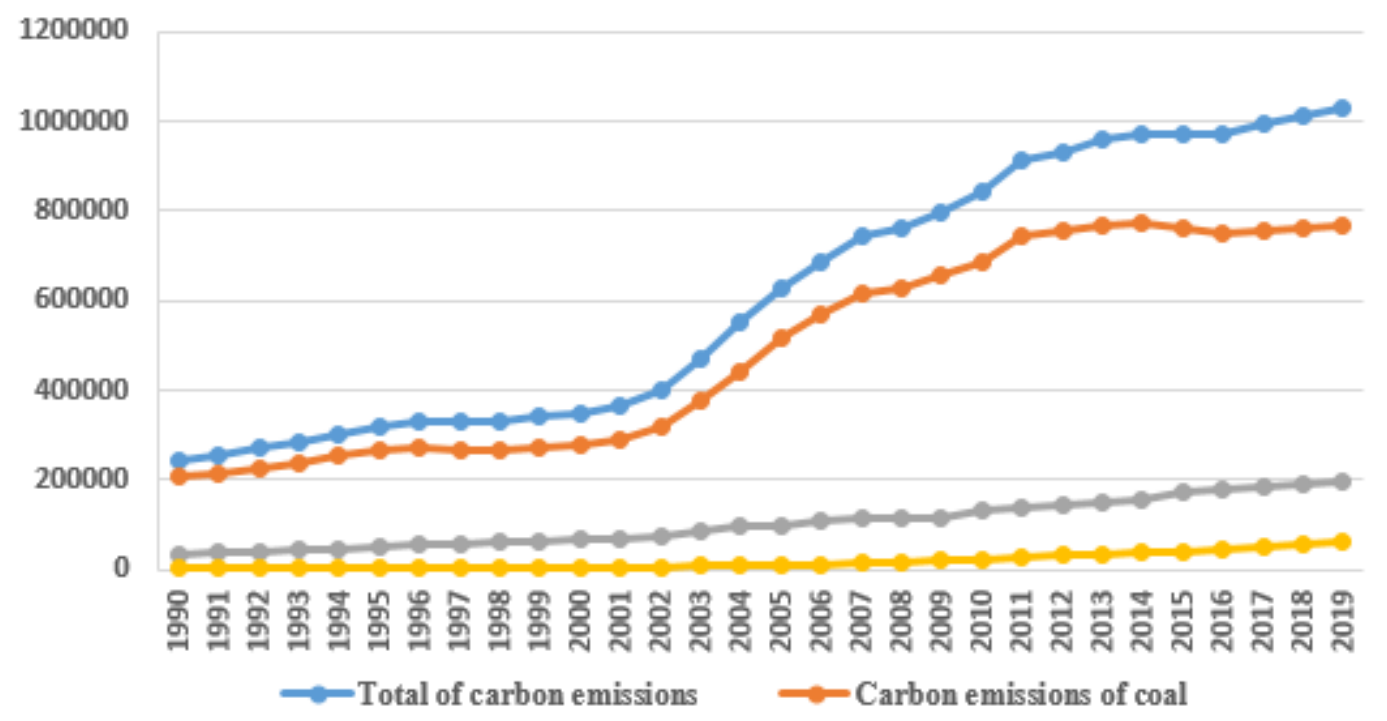

Figure 1

The trend of carbon dioxide emissions from China's energy consumption during 1990-2019 Unit: 10,000 tons
2. 0000
1. 8000
1. 6000
1. 4000
1. 2000
1. 0000
0.8000
0.6000
0.4000
0.2000
0.0000

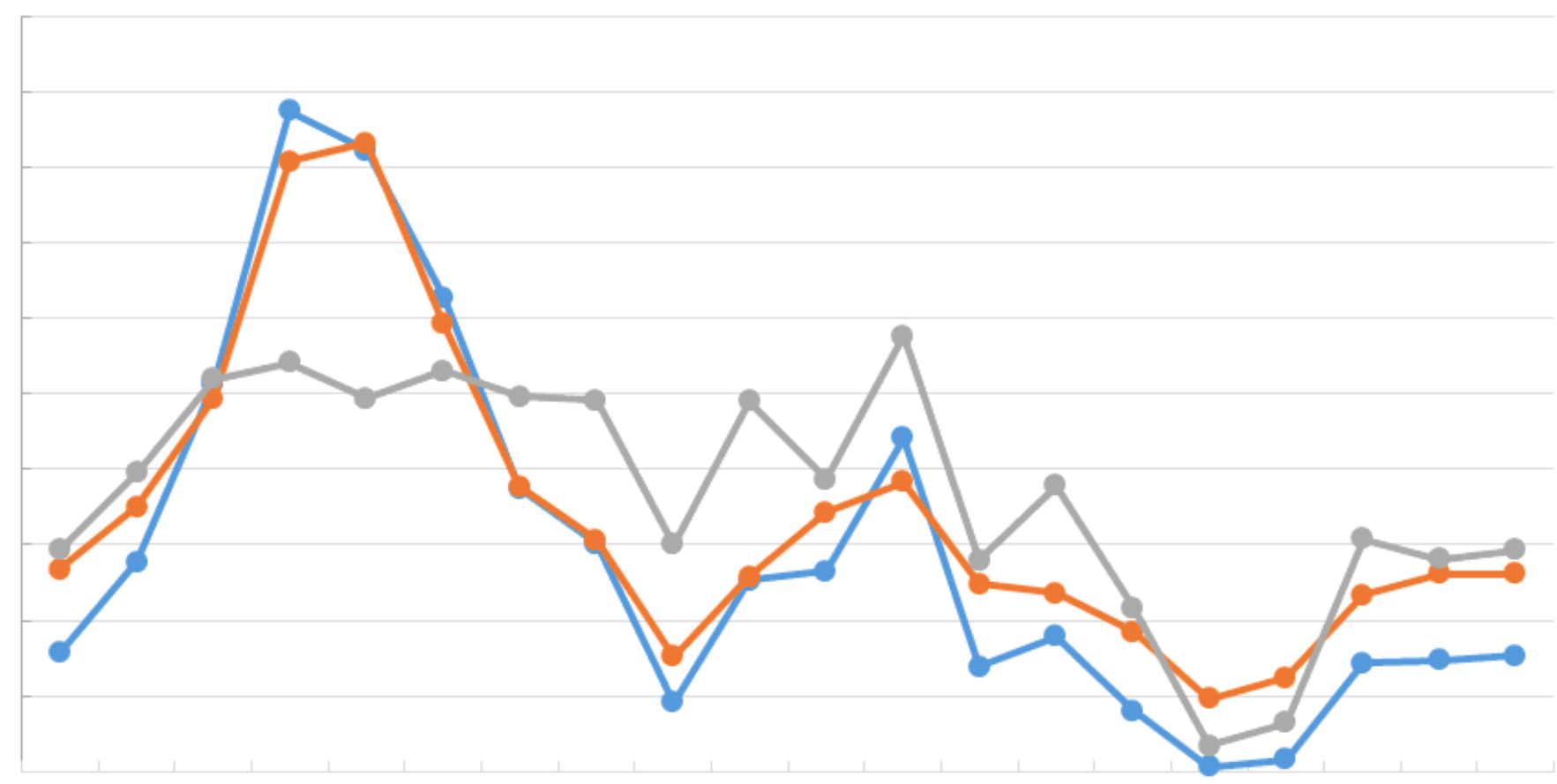

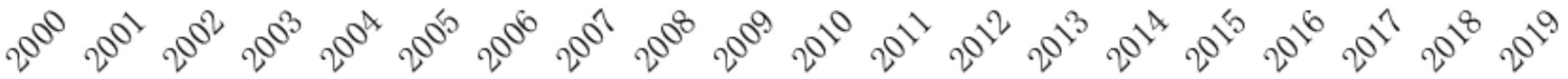

- The elastic decoupling index between carbon emissions and economic growth

- The elastic decoupling index between energy consumption and economic growth

- The elastic decoupling index between carbon emissions and energy consumption

Figure 2 
The elastic decoupling index between carbon emissions, energy consumption and economic growth from 2000 to 2019 